\title{
Effect of long-range interactions on the Kohn-Luttinger mechanism of the Cooper instability in the Shubin-Vonsowsky model
}

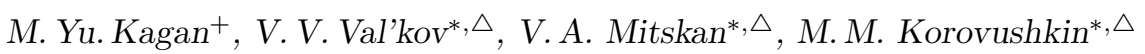 \\ ${ }^{+}$P. L. Kapitza Institute for Physical Problems, 119334 Moscow, Russia \\ ${ }^{*}$ L. V. Kirensky Institute of Physics, 660036 Krasnoyarsk, Russia \\ $\triangle_{\text {Siberian State Aerospace University, } 660014 \text { Krasnoyarsk, Russia }}$
}

Submitted 24 January 2013

\begin{abstract}
The problem of Cooper instability is studied by implementing the Kohn-Luttinger mechanism in the framework of the Shubin-Vonsowsky model taking into account the intersite Coulomb interactions within the first and second coordination spheres. It is shown that the Kohn-Luttinger renormalization for the effective interaction in the second-order terms of perturbation theory, as well as the inclusion of intersite hoppings in the second and third coordination spheres, significantly affects the interplay between the superconducting phases with $d_{x y}-, p-, s-$, and $d_{x^{2}-y^{2}}$-wave symmetries of the order parameter.
\end{abstract}

\section{INTRODUCTION}

The Kohn-Luttinger mechanism [1] attracts consid'erable current interest as a promising non-phonon mech'anism of superconductivity applicable for many physical 'systems such as cuprate superconductors [2] and doped graphene [3, 4, as well as the mechanism underlying superfluidity in ${ }^{3} \mathrm{He}$ [5] 7 and in topological superfluids [8]. The $d_{x^{2}-y^{2}}$-wave superconductivity arising in cuprates 'stimulated intense studies of the interplay between superconducting phases with different types of symmetry in the framework of the Hubbard model in the $U \ll W$ limit 9 14].

The effect of the screened Coulomb interaction on the Kohn-Luttinger mechanism for cuprate superconductors was recently studied in [15]. A renewed interest in the effect of the long-range Coulomb correlations on the structure of the phase diagrams for the MottHubbard systems at low electron density $n$ led to the ad'ditional studies [16, 17] based on the Shubin-Vonsowsky model 18, 19, which takes into account the interactions $(V)$ between electrons located at different lattice sites. In [17, the phase diagram in the $n-V$ plane was constructed for such a model. This phase diagram demonstrates the result of the interplay between superconducting phases with different types of symmetry. The corresponding calculations involve only the intersite hopping within the first coordination sphere and the intersite Coulomb interactions are considered including only the first-order terms of perturbation theory. Since the polarization Kohn-Luttinger contributions manifest themselves only in the second order, the analysis of the effects related to such contributions appears to be promising for finding out the ranges of existence for superconducting phases with different symmetries.

In this paper, the Cooper instability for the ShubinVonsowsky model is studied in the weak coupling limit of the Born approximation $(W>U>V)$ taking into account the long-range hopping processes and the intersite Coulomb interactions within the first and second coordination spheres. In the calculation of the effective interaction in the Cooper channel, we include the polarization contributions, graphically represented by four Kohn-Luttinger diagrams (Fig. 1). We demonstrate that the long-range Coulomb interactions and the long-range intersite electron hopping produce a pronounced effect on the conditions needed for the Cooper pairing with $s-, p-$, and $d$-wave symmetries of the order parameter. In particular, they illustrate the possibility of the $d_{x^{2}-y^{2}}$-type of pairing arising. Note that, in addition to the Shubin-Vonsowsky model, the $t-J$ model also remains a highly probable challenger for the adequate description of high- $T_{c}$ superconductivity with $d$-wave pairing 2023 . A consideration of the Coulomb interaction within the framework of the $t-J$ model will be a subject of our further studies.

\section{MODEL}

The Hamiltonian of the Shubin-Vonsowsky model in the quasimomentum representation has the form

$$
\begin{aligned}
\hat{H} & =\sum_{\mathbf{p} \sigma}\left(\varepsilon_{\mathbf{p}}-\mu\right) c_{\mathbf{p} \sigma}^{\dagger} c_{\mathbf{p} \sigma}+U \sum_{\mathbf{p} \mathbf{p} \mathbf{q}^{\prime}} c_{\mathbf{p} \uparrow}^{\dagger} c_{\mathbf{p}}^{\dagger}+\mathbf{q} \downarrow \\
& +\sum_{\mathbf{p} \mathbf{p} ' \mathbf{q} \sigma \sigma^{\prime}} V_{\mathbf{p}+\mathbf{q} \downarrow} c_{\mathbf{p}}{ }^{\prime} \uparrow \\
c_{\mathbf{p} \sigma}^{\dagger} c_{\mathbf{p}}^{\dagger}+\mathbf{q} \sigma^{\prime} & c_{\mathbf{p}+\mathbf{q} \sigma^{\prime}} c_{\mathbf{p} \sigma},
\end{aligned}
$$


where the electron energy including the long-range intersite hopping with the intensity determined by the parameters $t_{2}$ and $t_{3}$ is described by the expression

$$
\begin{aligned}
\varepsilon_{\mathbf{p}} & =2 t_{1}\left(\cos p_{x}+\cos p_{y}\right)+4 t_{2} \cos p_{x} \cos p_{y} \\
& +2 t_{3}\left(\cos 2 p_{x}+\cos 2 p_{y}\right) .
\end{aligned}
$$

The Fourier transform corresponding to the Coulomb repulsion between electrons located at the nearestneighbor or next-nearest-neighbor sites can be written as

$$
V_{\mathbf{p}}=2 V_{1}\left(\cos p_{x}+\cos p_{y}\right)+4 V_{2} \cos p_{x} \cos p_{y}
$$

The utilization of the aforementioned Born approximation in the weak-coupling limit allows us to use only the diagrams corresponding to the first and second orders of perturbation theory in terms of the coupling constant.

The opposite (strong-coupling) limit, $U>V>W$, was studied in [16. In this case, the inclusion of the first- and second-order diagrams is justified only in the low electron density limit $(n \ll 1)$, for which the Fermi gas type Galitskii-Bloom expansion [24,25] works well. Only the main exponential term for $T_{c}$ is calculated in this work, as well as in [16. The accurate evaluation of the pre-exponential factor requires the inclusion of the third and fourth-order diagrams. The Born approximation used in this paper allows us to consider also higher values of the electron density.

It is well known that the intersite Coulomb interaction suppresses the Cooper pairing in the first order of perturbation theory. The contributions to the effective interaction that improve the conditions favoring the Cooper instability appear in the second order. It is also important that the inclusion of the distant hopping provides an opportunity to shift the position of the Van Hove singularity in the electron density of states toward lower densities. In this paper, we analyze only the range of electron densities for which we do not approach too close to the Van Hove singularities, in order to avoid the summation of parquet diagrams 27 . In the Mott-Hubbard systems, the screening radius can exceed the unit cell size 28. This determines the efficiency of the Shubin-Vonsowsky model, which takes into account the intersite Coulomb interaction within several coordination spheres. In this case, the effects related to the Brillouin zone manifest themselves in the momentum dependence of $V_{\mathbf{p}}$, which is described by a periodic function, and are clearly pronounced.
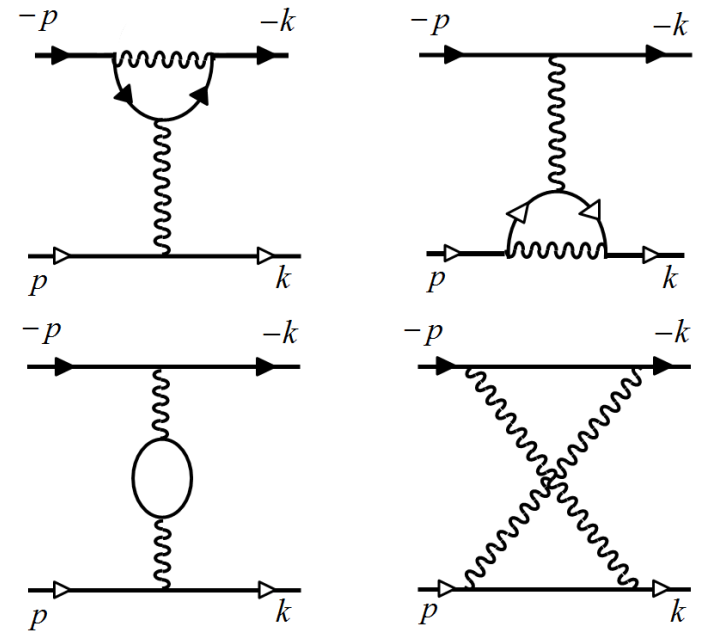

Fig. 1. Second-order diagrams for the effective interaction.

\section{RENORMALIZED INTERACTION IN THE COOPER CHANNEL}

The second-order correction $\delta \tilde{\Gamma}(p, k)$ for the effective interaction in the Cooper channel is determined by four Kohn-Luttinger diagrams shown in Fig. 1. Solid lines with the light (dark) arrows correspond to the Greens functions of electrons with spin projections equal to $+1 / 2(1 / 2)$. In these diagrams, the existence of two solid lines without arrows implies the performed summation over the values of the spin projections. The wavy lines correspond to the unrenormalized interactions. The scattering of electrons with the same spin projection gives rise only to the intersite contribution. If we have the interaction between electrons with opposite spins, the scattering amplitude is determined by the sum of the Hubbard and intersite interactions. Therefore, when we deal only with the Hubbard repulsion, the $\delta \tilde{\Gamma}(p, k)$ correction for the effective interaction is given only by the fourth diagram. If we take into account the Coulomb repulsion at the neighboring sites, all four diagrams shown in Fig. 1 contribute to the renormalized amplitude.

Introducing analytical expressions for the diagrams in Fig. 1 (by using four-vectors $p \equiv\left(\mathbf{p}, i \omega_{n}\right)$ and $k \equiv$ $\left.\left(\mathbf{k}, i \omega_{m}\right)\right)$ and performing the summation over the Matsubara frequencies, we arrive at the expression for the effective interaction

$$
\widetilde{\Gamma}(p, k)=U+V_{\mathbf{p}-\mathbf{k}}+\delta \widetilde{\Gamma}(p, k),
$$




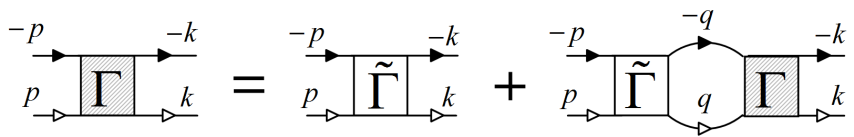

Fig. 2. Bethe-Salpeter equation.

$$
\begin{aligned}
& \delta \widetilde{\Gamma}(p, k)=\frac{1}{N} \sum_{\mathbf{p}_{1}}\left(U+V_{\mathbf{p}-\mathbf{k}}\right)\left(2 V_{\mathbf{p}-\mathbf{k}}-V_{\mathbf{p}_{1}+\mathbf{p}}-V_{\mathbf{p}_{1}-\mathbf{k}}\right) \\
& \times \frac{f\left(\varepsilon_{\mathbf{p}_{1}}\right)-f\left(\varepsilon_{\mathbf{p}_{1}+\mathbf{p}-\mathbf{k}}\right)}{i \omega_{n}-i \omega_{m}+\varepsilon_{\mathbf{p}_{1}}-\varepsilon_{\mathbf{p}_{1}+\mathbf{p}-\mathbf{k}}} \\
& +\frac{1}{N} \sum_{\mathbf{p}_{1}}\left(U+V_{\mathbf{p}_{1}-\mathbf{p}}\right)\left(U+V_{\mathbf{p}_{1}-\mathbf{k}}\right) \\
& \times \frac{f\left(\varepsilon_{\mathbf{p}_{1}}\right)-f\left(\varepsilon_{\mathbf{p}_{1}-\mathbf{p}-\mathbf{k}}\right)}{i \omega_{n}+i \omega_{m}-\varepsilon_{\mathbf{p}_{1}}+\varepsilon_{\mathbf{p}_{1}-\mathbf{p}-\mathbf{k}}},
\end{aligned}
$$

where $f(\varepsilon)=\left(\exp \left(\frac{\varepsilon-\mu}{T}\right)+1\right)^{-1}$.

\section{BETHE-SALPETER EQUATION}

Utilizing the renormalized interaction in the weakcoupling approximation, we get the ladder-type equation for the scattering amplitude in the Cooper channel (see Fig. 2). In the explicit form, this equation reads as

$$
\begin{aligned}
& \Gamma(p \mid k)=\widetilde{\Gamma}(p \mid k) \\
& -\frac{T}{N} \sum_{\mathbf{q}, \omega_{l}} \widetilde{\Gamma}\left(p \mid \mathbf{q}, i \omega_{l}\right) \frac{\Gamma\left(\mathbf{q}, i \omega_{l} \mid k\right)}{\left(i \omega_{l}-\xi_{\mathbf{q}}\right)\left(-i \omega_{l}-\xi_{\mathbf{q}}\right)},
\end{aligned}
$$

where $\xi_{\mathbf{q}}=\varepsilon_{\mathbf{q}}-\mu$. Here, performing the summation over the Matsubara frequencies $\omega_{l}$, we take into account that the main contribution to the sum in the Cooper channel in the weak-coupling approximation comes from the $\omega_{l}$ values close to zero. Therefore, we can neglect the frequency dependence of the renormalized interaction. In this case, the total amplitude is independent of the frequency and the summation over $\omega_{l}$ in the BetheSalpeter equation can be performed explicitly. As a result, we get the following integral equation, which determines the momentum dependence of the scattering amplitude

$\Gamma(\mathbf{p} \mid \mathbf{k})=\widetilde{\Gamma}(\mathbf{p} \mid \mathbf{k})-\frac{1}{N} \sum_{\mathbf{q}} \widetilde{\Gamma}(\mathbf{p} \mid \mathbf{q}) \frac{\tanh \left(\frac{\xi_{\mathbf{q}}}{2 T}\right)}{2 \xi_{\mathbf{q}}} \Gamma(\mathbf{q} \mid \mathbf{k}) .(6)$

It is well known that the pole corresponding to the Cooper instability can be found analyzing the homogeneous part of the reduced equation [29]. Introducing the integration over the isoenergetic curves and taking into account that the main contribution comes from the contours close to the Fermi contour [10-12, 15], we get the equation

$$
\frac{1}{(2 \pi)^{2}} \oint_{\varepsilon_{\mathbf{q}}=\mu} \frac{d \hat{\mathbf{q}}}{v_{F}(\hat{\mathbf{q}})} \widetilde{\Gamma}(\hat{\mathbf{p}} \mid \hat{\mathbf{q}}) \Gamma(\hat{\mathbf{q}})=\lambda \Gamma(\hat{\mathbf{p}}),
$$

where $\lambda^{-1} \simeq \ln \left(T_{c} / W\right)$, quasimomenta $\hat{\mathbf{p}}$ and $\hat{\mathbf{q}}$ lie on the Fermi surface, $v_{F}(\hat{\mathbf{q}})$ is the Fermi velocity. To solve this equation, we should consider an eigenvalue problem.

We represent the kernel of integral equation (7) as a superposition of the functions, each belonging to one of the irreducible representations of the $C_{4 v}$ symmetry group on the square lattice. It is well known that this group has five irreducible representations 30. For each representation, Eq. (7) has a solution with its own effective coupling constant $\lambda$. Further on, we use the following notation to classify the symmetries of the order parameter: representations $A_{1}, A_{2}, B_{1}, B_{2}$, and $E$ correspond to the $s$-wave, extended $s$-wave, $d_{x y}$-wave, $d_{x^{2}-y^{2}}$-wave, and $p$-wave types of symmetry, respectively.

We seek a solution of Eq. (77) in the form

$$
\Gamma(\phi)=\sum_{\alpha n} \Delta_{\alpha n} g_{\alpha n}(\phi),
$$

where $\alpha$ is the ordinal number of the representation, $n$ is the ordinal number of a function belonging to the given representation, and $\phi$ is the angle characterizing the direction of the quasimomentum $\hat{\mathbf{p}}$ with respect to the $p_{x}$ axis. The explicit form of the orthonormalized functions $g_{\alpha n}(\phi)$ is given by the expressions

$$
\begin{aligned}
& A_{1} \rightarrow g_{s, n}(\phi)=\frac{1}{\sqrt{\left(1+\delta_{n 0}\right)}} \cos 4 n \phi, \quad n \in[0, \infty) \\
& A_{2} \rightarrow g_{s_{e x t}, n}(\phi)=\frac{1}{\sqrt{\pi}} \sin 4(n+1) \phi \\
& B_{1} \rightarrow g_{d_{x y}, n}(\phi)=\frac{1}{\sqrt{\pi}} \sin (4 n+2) \phi \\
& B_{2} \rightarrow g_{d_{x^{2}-y^{2}}, n}(\phi)=\frac{1}{\sqrt{\pi}} \cos (4 n+2) \phi \\
& E \rightarrow g_{p, n}(\phi)=\frac{1}{\sqrt{\pi}}(A \sin (2 n+1) \phi+B \cos (2 n+1) \phi)
\end{aligned}
$$

Substituting Eq. (8) into Eq. (7), performing integration over the angles, and using the orthonormality condition for the functions $g_{\alpha n}(\phi)$, we find

$$
\hat{\Lambda}_{\alpha n ; \beta m} \Delta_{\beta m}=\lambda \Delta_{\alpha n},
$$

where

$$
\begin{aligned}
\hat{\Lambda}_{\alpha n ; \beta m} & =\frac{1}{(2 \pi)^{2}} \int_{0}^{2 \pi} d \phi_{\mathbf{p}} \int_{0}^{2 \pi} d \phi_{\mathbf{q}} \frac{d \hat{\mathbf{q}}}{d \phi_{\mathbf{q}} v_{F}(\hat{\mathbf{q}})} \widetilde{\Gamma}(\hat{\mathbf{p}} \mid \hat{\mathbf{q}}) \\
& \times g_{\alpha n}\left(\phi_{\mathbf{p}}\right) g_{\beta m}\left(\phi_{\mathbf{q}}\right) .
\end{aligned}
$$



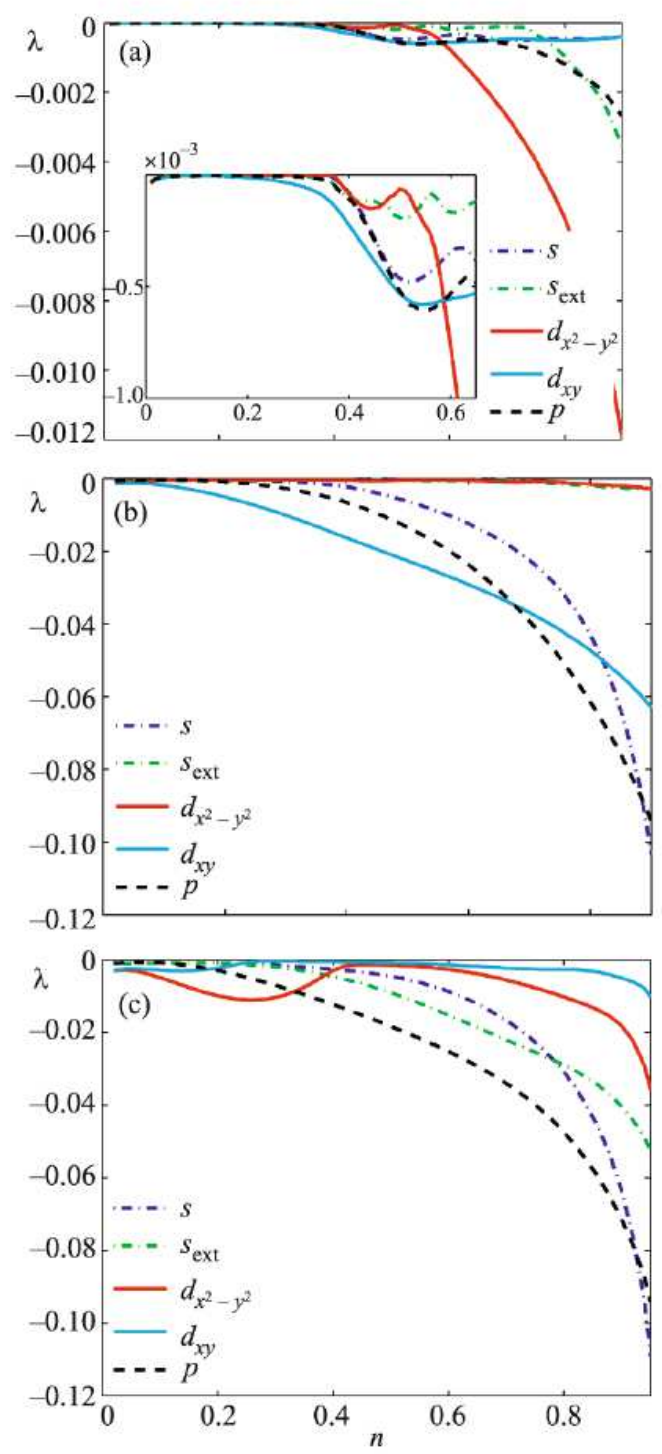

Fig. 3. Electron density dependence of $\lambda$ for $t_{2}=$ $t_{3}=0$ and $U=1$ at different values of the parameters characterizing the intersite Coulomb interaction: (a) $V_{1}=V_{2}=0$; (b) $V_{1}=0.5, V_{2}=0$; (c) $V_{1}=0.5, V_{2}=0.25$.

Since $T_{c} \sim \exp (1 / \lambda)$, each negative eigenvalue $\lambda$ corresponds to a superconducting phase with the specified symmetry type of the order parameter. Each solution corresponds to only one irreducible representation, but its expansion in terms of the basis functions generally includes several angular harmonics. The highest critical temperature corresponds to the largest absolute value of $\lambda$.

\section{RESULTS AND DISCUSSION}

In Fig. 3, we illustrate the dependence of the effective coupling constant $\lambda$ on the electron density $n$ for different types of symmetry of the superconducting order parameter. The calculations were performed at $t_{2}=t_{3}=0$ and $U=1$ (all energy parameters are measured in units of $\left|t_{1}\right|$ ) at different values of the parameters $V_{1}$ and $V_{2}$, which characterize the intersite Coulomb interactions. In Fig. 3 (a), we plot the $\lambda(n)$ curve in the absence of the intersite Coulomb interaction $\left(V_{1}=0\right.$ and $V_{2}=0$ ). It agrees well with the corresponding curves reported in 11]. At low electron densities $(n=0-0.52)$, in the first two orders of perturbation theory, we get superconductivity with the $d_{x y}$-wave type of the order parameter [10]. In the range $n=0.52-0.58$, the ground state corresponds to the phase with the $p$-wave order parameter. At $n>0.58$, the $d_{x^{2}-y^{2}}$-wave type of superconductivity is the dominant one.

The inclusion of the intersite Coulomb interaction significantly affects the interplay between the superconducting phases. This is clearly seen in Fig. 3 (b), where we represent the $\lambda(n)$ plots for $V_{1}=0.5$ and $V_{2}=0$. Their comparison to the plots in Fig. 3 (a) demonstrates that the range of dominance for the $d_{x y}-$ and $p$-wave phases becomes broader. At high electron densities $(n \geq 0.89)$, we have the $s$-wave type of pairing, for which the main contribution comes from the $g_{s, 1}(\phi)=\frac{1}{\sqrt{\pi}} \cos 4 \phi$ harmonics.

It is necessary to note that the Cooper pairing calculated up to the second order of perturbation theory in terms of the Coulomb interaction is not suppressed by the first-order terms. This stems from the fact that the bare Coulomb repulsion $V_{\mathbf{p}-\mathbf{k}}$ suppresses only a part of the harmonics, whereas the other harmonics lead to the Cooper instability. For example, in the range of existence for the $p$-wave pairing, the main contribution of the bare repulsion $V_{\mathbf{p}-\mathbf{k}}$ in the $p$-wave channel is given by the function $g_{p, 0}(\phi)=$ $\frac{1}{\sqrt{\pi}}(A \sin \phi+B \cos \phi)$, whereas the main contribution to the scattering amplitude in the $p$-channel comes from the function $g_{p, 1}(\phi)=\frac{1}{\sqrt{\pi}}(A \sin 3 \phi+B \cos 3 \phi)$.

In Fig. 3 (c), we represent the $\lambda(n)$ curves plotted taking into account the intersite Coulomb interactions within the first and second coordination spheres $\left(V_{1}=0.5\right.$ and $\left.V_{2}=0.25\right)$. The comparison with Fig. [3 (b) suggests that the inclusion of the long-range Coulomb repulsion $V_{2}$ favors the $d_{x^{2}-y^{2}}$-wave type of pairing at low charge carrier densities $(n=0.05-0.34)$.

Distant electron hoppings (to the sites located out- 

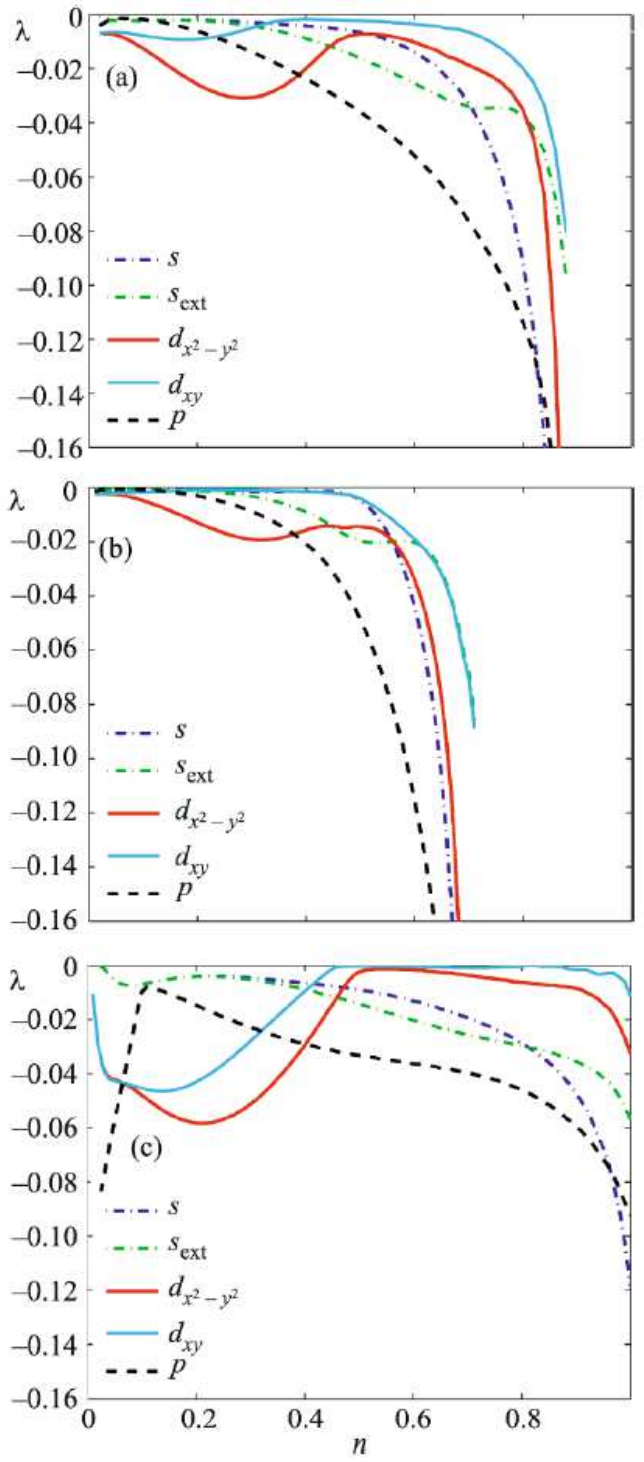

Fig. 4. Electron density dependence of $\lambda$ for $U=1$; $V_{1}=0.5, \quad V_{2}=0.25$ at different values of the distant hopping integrals: (a) $t_{2}=0.15, t_{3}=0$; $t_{2}=0.15, t_{3}=-0.1 ; \quad$ (c) $t_{2}=0.15, t_{3}=0.1$.

side the first coordination sphere) significantly affect the interplay of different superconducting phases. This is illustrated in Fig. 4, where we show the $\lambda(n)$ curves plotted at $U=1, V_{1}=0.5$, and $V_{2}=0.25$ for different values of $t_{2}$ and $t_{3}$. The plots shown in Fig. 田(a) are calculated with the inclusion of the electron hoppings within the first two coordination spheres $\left(t_{2}=0.15, t_{3}=0\right)$. At these parameters, the critical density of fermions $n_{v H}$ (corresponding to the Van Hove singularity) shifts from the half filling toward lower electron densities (Fig. (5). Comparing Figs. 3 (c) and 4 (a), we see that the inclusion of the distant hoppings $t_{2}$ leads to broadening of

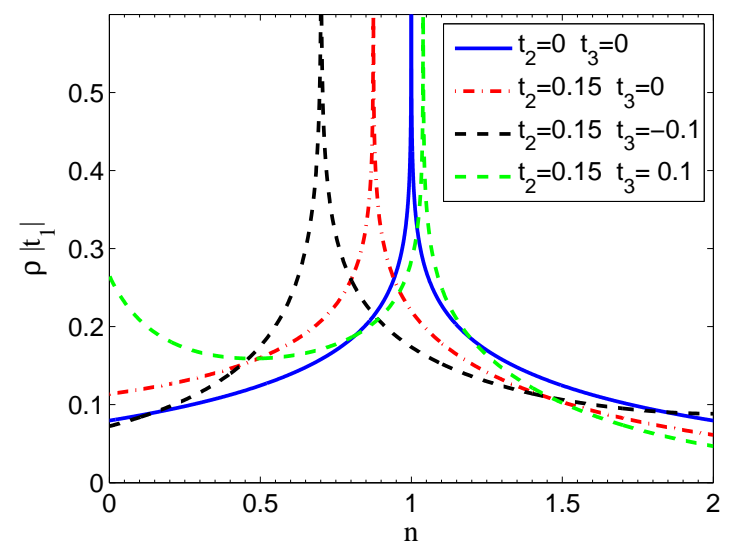

Fig. 5. Evolution of the electron density of states under variation of the hopping integrals.

the dominance range for the $d_{x^{2}-y^{2}}$-type of pairing to $n=0.4$ and to an increase in the absolute value of $\lambda$ in this region.

Figures 4 (b) and 4 (c) show the $\lambda(n)$ dependence calculated with the additional inclusion of hoppings to the third coordination sphere with $t_{3}<0$ and $t_{3}>0$, respectively. Comparison of these plots indicates that the inclusion of hoppings with $t_{3}>0$ leads to the additional broadening of the dominance range for the $d_{x^{2}-y^{2}}$-wave type of pairing at low charge carrier densities and to the enhancement of the effective interaction in this region.

Note that with the growth of $U$, the superconducting phase with the $d_{x^{2}-y^{2}}$-wave symmetry of the order parameter becomes dominant in the density range close to the Van Hove singularities. This can be seen in Fig. 6, where we demonstrate the $\lambda(n)$ curves calculated at $U=2$. In the regions of low and high densities, the $d_{x^{2}-y^{2}}$ phase corresponds to the ground state of the system. This result seems to be important for the analysis of the mechanisms underlying the high- $T_{c}$ superconductivity. Note in this context that the critical temperatures $T_{c} \sim 100 \mathrm{~K}$ appear at $U=3$. However, this case is on the verge of the applicability range of the weak-coupling approximation, which we use in this paper.

\section{CONCLUSIONS}

The analysis of the Cooper instability performed within the framework of the Shubin-Vonsowsky model in the weak-coupling limit $(W>U>V)$ demonstrates that the Kohn-Luttinger contributions of the polarization type calculated up to the second order of perturbation theory lead to a significant renormalization of 


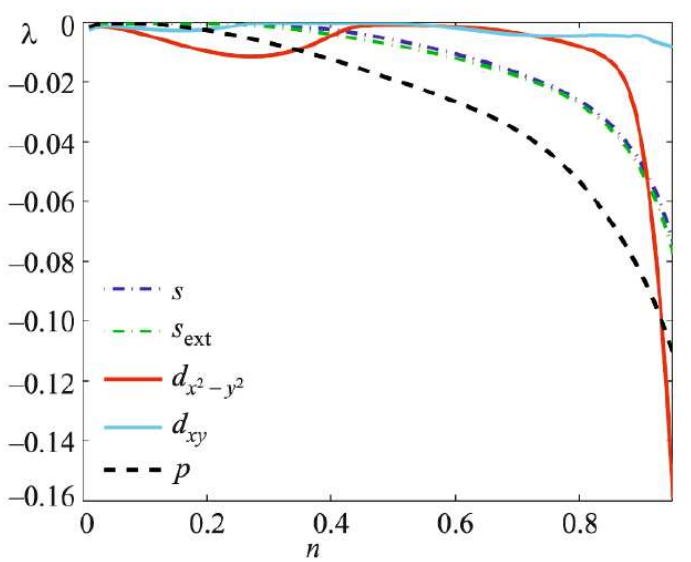

Fig. 6. Carrier density dependence of $\lambda(n)$ at $t_{2}=t_{3}=$ $0, V_{1}=0.5, V_{2}=0.25$ and $U=2$.

the effective interaction. Note that the inclusion of the additional Coulomb repulsion at the neighboring lattice sites $\left(V_{1}\right)$ and even at the next-nearest-neighbor sites $\left(V_{2}\right)$ cannot suppress all superconducting channels in the calculations of the effective interaction $\tilde{\Gamma}$ in the first two orders of perturbation theory.

At $V_{1} \neq 0$ and $V_{2} \neq 0$ superconductivity arises in the $p-, d_{x y}-$, and $d_{x^{2}-y^{2}}-$ wave channels at low and intermediate electron densities. In the $d_{x^{2}-y^{2}}$-wave and in the specific $s$-wave channel ( $\cos 4 \phi$ harmonics), it arises near the Van Hove singularity at high electron densities. Near the Van Hove singularity, with the growth of the Hubbard repulsion $U$, we get the usual $d_{x^{2}-y^{2}}$-wave type of pairing typical of cuprates with rather realistic values of the superconducting transition temperature. The inclusion of the distant hoppings $t_{2} \neq 0$ and $t_{3} \neq 0$ shifts the Van Hove singularity toward lower electron densities but does not change the gross phase diagram describing the superconducting states.

We are grateful to A. S. Alexandrov, D. V. Efremov, V. V. Kabanov, Yu. V. Kopaev, K. I. Kugel, M. S. Marenko, N. M. Plakida, and A.V. Chubukov for numerous fruitful discussions and permanent interest in this work. This work was supported by the Presidium of the Russian Academy of Sciences (program no. 20.7 "Quantum Mesoscopic and Disordered Structures"), by the Russian Foundation for Basic Research (project nos. 11-02-00741 and 12-02-31130), and by the Ministry of Education and Science of the Russian Federation (state contract no. 16.740.11.0644, federal program "Human Capital for Science and Education in Innovative Russia" for 20092013). M. M. K. acknowledges the support of the Council of the President of the Russian Federation for Support of Young Scientists and Leading Scientific
Schools (project no. MK-526.2013.2) and the Dynasty Foundation.

1. W. Kohn and J. M. Luttinger, Phys. Rev. Lett. 15, 524 (1965).

2. M. Yu. Kagan and A. V. Chubukov, JETP Lett. 47, 614 (1988); 50, 517 (1989); M. A. Baranov, A. V. Chubukov, and M. Yu. Kagan, Int. J. Mod. Phys. B 6, 2471 (1992).

3. J. González, Phys. Rev. B 78, 205431 (2008).

4. R. Nandkishore, L. S. Levitov, and A. V. Chubukov, Nature Phys. 8, 158 (2012).

5. M. Yu. Kagan, Phys. Usp. 37, 69 (1994).

6. D. Vollhardt and P. Wolfle, The superfluid phases of Helium 3, Taylor and Francis, London, 1990.

7. G. E. Volovik, The Universe in a Helium Droplet, Oxford University Press, 2003, 536 pp.; G. E. Volovik, Exotic properties of superfluid 3He, World Scientific, 1992.

8. M.S. Marienko, J.D. Sau, and S. Tewari, arXiv:1202.5784v1 (2012).

9. M. Yu. Kagan, Phys. Lett. A 152, 303 (1991).

10. M. A. Baranov and M. Yu. Kagan, Z. Phys. B - Condens. Matter 86, 237 (1992).

11. R. Hlubina, Phys. Rev. B 59, 9600 (1999); J. Mráz and R. Hlubina, Phys. Rev. B 67, 174518 (2003).

12. S. Raghu, S.A. Kivelson, and D. J. Scalapino, Phys. Rev. B 81, 224505 (2010); S. Raghu and S. A. Kivelson, Phys. Rev. B 83, 094518 (2011).

13. D. J. Scalapino, E. Loh, Jr., and J. E. Hirsch, Phys. Rev. B 34, 8190 (1986)

14. D. Zanchi and H. J. Schulz, Phys. Rev. B 54, 9509 (1996)

15. A.S. Alexandrov and V. V. Kabanov, Phys. Rev. Lett. 106, 136403 (2011).

16. M. Yu. Kagan, D. V. Efremov, M. S. Marienko, and V. V. Val'kov, JETP Lett. 93, 819 (2011).

17. S. Raghu, E. Berg, A. V. Chubukov, and S. A. Kivelson, Phys. Rev. B 85, 024516 (2012).

18. S. Shubin and S. Vonsowsky, Proc. Roy. Soc. A 145, 159 (1934).

19. S. Shubin and S. Vonsowsky, Phys. Zs. UdSSR 7, 292 (1935); 10, 348 (1936).

20. M. Yu. Kagan and T. M. Rice, J. Phys.: Condens. Matter 6, 3771 (1994).

21. N. M. Plakida, L. Anton, S. Adam, and G. Adam, JETP 97, 331 (2003); N. M. Plakida, JETP Lett. 74, 36 (2001).

22. Yu. A. Izyumov, Phys. Usp. 42, 215 (1999).

23. V.I. Belinicher, A.L. Chernyshev, and V.A. Shubin, Phys. Rev. B 56, 3381 (1997); V. I. Belinicher, A. L. Chernyshev, A. V. Dotsenko, and O. P. Sushkov, Phys. Rev. B 51, 6076 (1995).

24. V. M. Galitskii, Sov. Phys. JETP 7, 104 (1958).

25. P. Bloom, Phys. Rev. B 12, 125 (1975). 
26. D. V. Efremov, M. S. Marenko, M. A. Baranov, M. Yu Kagan, JETP 90861 (2000).

27. I. E. Dzyaloshinskii and V. M. Yakovenko, Sov. Phys. JETP 67 (4), 844 (1988).

28. R. O. Zaitsev, JETP 98, 780 (2004).

29. L. P. Gorkov and T. K. Melik-Barkhudarov, Sov. Phys. JETP 13, 1018 (1961).

30. L. D. Landau and E. M. Lifshitz, Course of Theoretical Physics, Vol. 3: Quantum Mechanics: Non-Relativistic Theory (Nauka, Moscow, 1989, 4th ed.; Pergamon, New York, 1977, 3rd ed.). 